\title{
Pre- and Post-Operative Volume Estimation of Medulloblastoma Using Magnetic Resonance Imaging: A Stereological Study
}

\author{
Farzaneh Dehghani ${ }^{1,2}$, Seyede Fatemeh Hosseini ${ }^{1,2,{ }^{*}}$, Reza Jalli ${ }^{3}$, Fatemeh Karimi ${ }^{1,2}$, Narges \\ Sotoudeh ${ }^{1,2}$ and Mohammad Sadegh Masoudi ${ }^{4}$ \\ ${ }^{1}$ Histomorphometry and Stereology Research Center, Shiraz University of Medical Sciences, Shiraz, Iran \\ ${ }^{2}$ Anatomy Department, Shiraz University of Medical Sciences, Shiraz, Iran \\ ${ }^{3}$ Medical Imaging Research Center, Shiraz University of Medical Sciences, Shiraz, Iran \\ ${ }^{4}$ Neurosurgery Department, Shiraz University of Medical Sciences, Shiraz, Iran \\ "Corresponding author: Anatomy Department, Shiraz University of Medical Sciences, Shiraz, Iran. Tel: +98-9910826194, Email: shosseini2020.15@gmail.com
}

Received 2019 August 11; Revised 2020 April 26; Accepted 2020 May 02.

\begin{abstract}
Background: Brain tumors are among the most lethal and devastating cancers. Medulloblastoma tumor is a common solid brain malignancy, arising in the posterior fossa. Stereological methods are used in combination with magnetic resonance imaging (MRI) to obtain unbiased estimation of the total volume of the structure of interest.

Objectives: In the current study, we evaluated cerebellar tumor volume pre- and post-operative in patients suffering from meduloblastoma tumor using MRI and Cavalieri method.

Patients and Methods: The study was performed on 16 subjects consisting of two groups of eight people including patient and control groups. Slide direction were in both sagittal and axial planes. Pre- and post-operative volume of medulloblastoma tumor were determined on MR scanning images using the point-counting approach of Cavalieri method.

Results: The post-operative tumor volume in both sagittal $(\mathrm{P}=0.028)$ and axial $(\mathrm{P}=0.046)$ MR images was statistically reduced by $90 \%$ compared to the pre-operative volume. Moreover, the pre-operative volume of the cerebellum in patients increased by $38 \%$ in patients as compared to the normal subjects $(\mathrm{P}=0.028)$.

Conclusion: It could be concluded that MRI-estimated tumor-volume might be useful in evaluating the efficiency of surgical treatment and prognostication of tumor regression rate.
\end{abstract}

Keywords: Meduloblastoma, Cavalieri method, MRI

\section{Background}

In recent years, numerous studies have been conducted on brain tumors. The cerebellar tumors are often known as infratentorial or posterior fossa tumors (1). Posterior fossa tumors constitute $75 \%$ of all pediatric brain tumors (1-3). Pilocytic astrocytoma, a low grade glial tumor, is the most common pediatric central nervous system (CNS) tumor, accounting for about 35\% of all diagnoses $(4,5)$. Infratentorial ependymoma includes $15 \%$ of posterior fossa tumors in children and is more common in males than females $(6,7)$. Atypical teratoid rhabdoid tumors (ATRTs) are infrequent and highly aggressive CNS tumors that primarily occur in infants less than 3 years of age $(8,9)$. Medulloblastoma (MB) is a common malignant ectodermal tumor accounting for 7 to $15 \%$ of all brain tumors $(10,11)$. Based on World Health Organization (WHO) classification, medulloblastoma is subdivided into five major groups: classic, anaplastic, large cell, desmoplastic, and extensive nodularity subtypes (12). The mean overall incidence of MB is estimated at approximately six children per million and the survival rate is $50 \%$ to $65 \%$ for 5 -years (1012). Treatment strategies for this malignant neoplasm include a combination of surgery, chemotherapy, and following radiotherapy (13).

Recently, quantitative techniques, such as computed tomography (CT) scans or magnetic resonance imaging (MRI), is developing for effective volume measuring of a structure with arbitrary shape and size (14). MRI is the best imaging modality in the diagnosis of intracranial tumors due to its high soft tissue contrast and multiplane capability. On the other hand, under a proper sampling design, stereological methods may be applied to estimate geometric parameters of any object $(14,15)$. For example, the Cavalieri method is used in combination with point counting to obtain an assumption-free estimate of the total volume 
of the structure of interest (16). The method is delivered by the standard software can be tested and so assessed with Cavalieri methods for brain volume estimation. Liu et al. (2007), Ertl-Wagner et al. (2009) and Gong et al. (2004) evaluated the tumor volume using MRI and stereological methods (16-18).

\section{Objectives}

In the current study, we evaluated and compared the cerebellum and tumor volume in patients suffering from meduloblastoma tumor, using MRI and Cavalieri stereological method.

\section{Patients and Methods}

\subsection{Patients}

The current study was performed between December 2015 and August 2018. The study was carried out on 16 subjects ( $4-7$ years old) consisting of two groups of eight people (five female and three male) including patients (with medulloblastoma tumor) and control (five female and three male) with a mean age of 5.7 years. Diagnosis of all patients was based on pathology and imaging findings. Metastatic and vascular tumors were eliminated from the study. In this regard, WHO criteria for pathological diagnosis was used. All the participants' parents signed the consent forms.

\subsection{MRI Procedure}

An MRI was performed hours before surgery. An identical MRI was obtained from the patients 2 weeks after surgery. In the pre- and post-operation time, contrast sagittal and axial T1-weighted imaging as well as T2 weighted MR sectioning and fluid-attenuated inversion recovery (FLAIR) sequence imaging were performed for all patients using a 1.5 Tesla MR machine (Philips, Intera, The Netherlands). The following parameters were used for the $\mathrm{T} 1$ imaging slices: 2 signal averaging number, repetition time/echo time (TR/TE): $25 / 5$, field of view (FOV): $130 \times 160,0.7 \mathrm{~mm}$ slice thickness (ST) with no interval between the sections, and $224 \times 224$ matrix.

\subsection{Cavalieri Principle Applied to MRI Sections}

Stereological methods, such as Cavalieri method, were used to obtain an unbiased volume estimation of a structure of arbitrary shape and size effectively. According to Cavalieri principle, the MRIs of a section series with a thickness of $0.7 \mathrm{~mm}$ were used to estimate the structures and tumor volume. The square grid test system with $\mathrm{d}=0.4 \mathrm{~cm}$ between test points was superimposed on MRI films (Figure 1). The points hitting the structure sectioned surface area were counted for each section and the volume of the cerebellum and $\mathrm{MB}$ tumor were estimated, using the following formula (Equation 1):

$$
(V)=t \times(a / p) \times\left[\sum P-\frac{1}{2} P \max \right]
$$

$$
\begin{aligned}
& C E=\left(\sum P\right) \\
& -1 \\
& \times\left[1 / 2\left(3 \times \sum P i P i+\sum P i P i+2-4 \sum P i P i+1\right) C E(V)\right. \\
& =\left(\sum P\right)-1 \times\left[1 / 2\left(3 \times \sum P i P i+\sum P i P i+2-4 \sum P i P i+1\right)\right. \\
& \left.+0.0724 \times b /(a 1 / 2) \times\left(n \sum P i\right) 1 / 2\right] 1 / 2
\end{aligned}
$$

where $t$ is the section thickness, a/p represents the area of each point on the point counting grid, $\sum \mathrm{P}$ is the total number of the points hitting the section's cut surface, and $1 / 2$ Pmax is half of the maximal number of points counted on the largest section of the examined subject $(14,15)$.

\subsection{Statistical Analysis}

The volume of the interested structures was compared between the patients and normal subjects using MannWhitney $U$ test. The volume in intervention group during pre and post of surgery were compared by Wilcoxon signed-rank test. The volume data are expressed in mean \pm standard deviation (SD). All P values $<0.05$ were measured as statistically significant. All statistical analysis were performed using GraphPad prism software (version 8) and SPSS software (IBM Corp. Released 2013. IBM SPSS Statistics for Windows, Version 22.0. Armonk, NY: IBM Corp.).

\section{Results}

The volume of the brain and cerebellum in both axial and sagittal MR planes in the normal and patient groups are shown in Table 1. Cerebellar volume was significantly increased in patients compared to healthy controls. Postoperative cerebellar volume did not show a significant difference with the controls and was very close to the normal values.

The volume of the brain, cerebellum, and MB tumor in both axial and sagittal MR planes in the pre- and post- operation situation in the patient group are shown in Table 2.

The volume of the MB tumor in both axial and sagittal MR planes showed significant decreases in the post- operation time compared to pre-operation value. Furthermore, the volume of the cerebellum in axial MR planes showed 

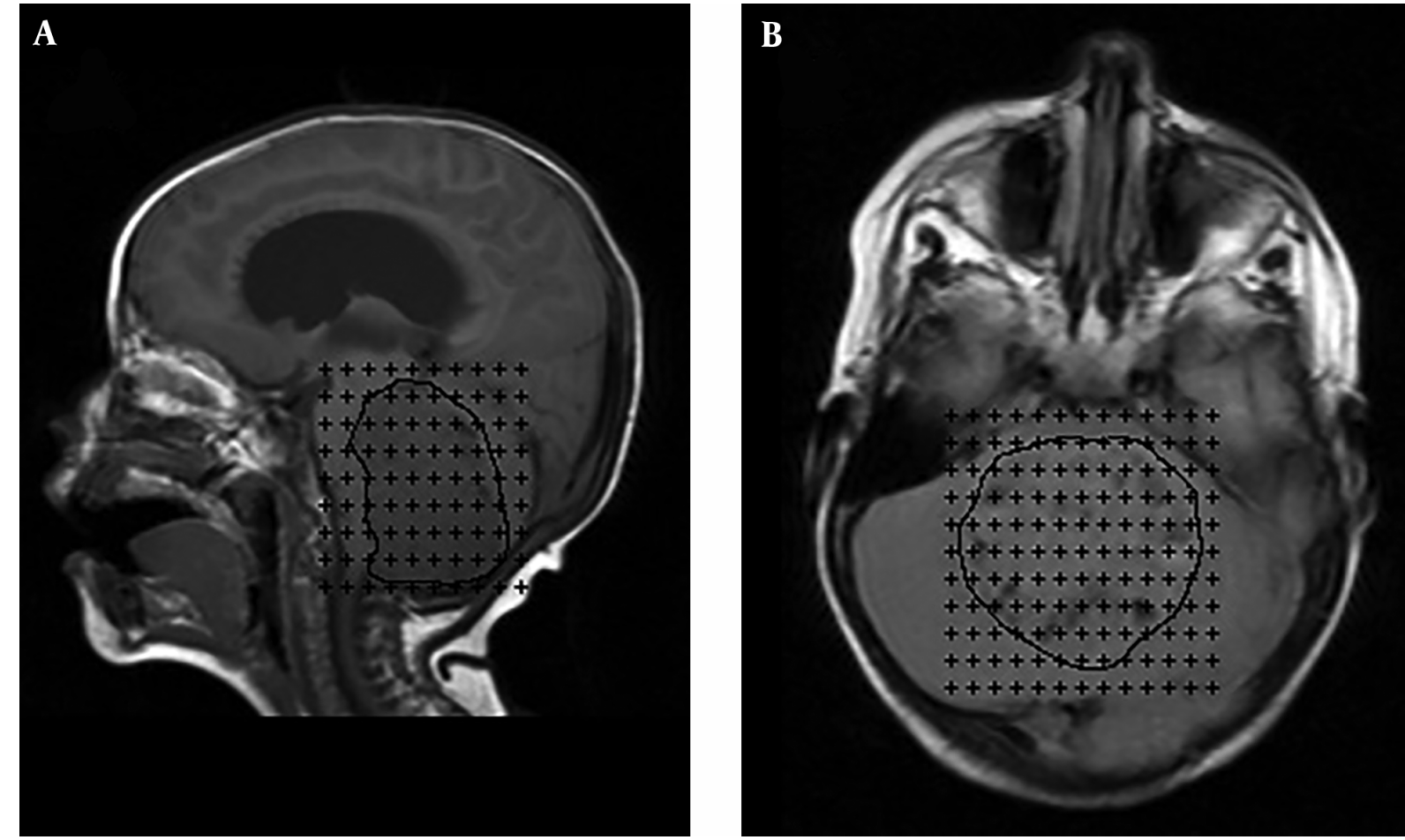

Figure 1. Sagittal (A) and axial (B) MR scans with a point-counting grid overlaid on images for the estimation of tumor volume using the Cavalieri technique. The border of the tumor is determined with a black line. Each point $(+)$ is associated with a known area $(\mathrm{a} / \mathrm{p})$. (a/p; represents the area of each point on the point counting grid)

\begin{tabular}{|c|c|c|c|c|c|}
\hline \multirow{2}{*}{ Parameters } & \multirow{2}{*}{ Normal group } & \multicolumn{4}{|c|}{ Patient group } \\
\hline & & Pre operation & Pvalue & Post operation & P value \\
\hline Brain volume, $\mathrm{cm}^{3}$ (axial MR images) & $1350.69 \pm 158.49$ & $1475.62 \pm 177.17$ & 0.645 & $1282.52 \pm 157.09$ & 0.645 \\
\hline Brain volume, $\mathrm{cm}^{3}$ (sagittal MR images) & $1478.41 \pm 147.64$ & $1471.14 \pm 150.17$ & 0.936 & $1273.91 \pm 170.37$ & 0.367 \\
\hline Cerebellar volume, $\mathrm{cm}^{3}$ (axial MR images) & $158.09 \pm 20.08$ & $236.81 \pm 27.82$ & $0.037^{*}$ & $161.74 \pm 22.22$ & 0.959 \\
\hline Cerebellar volume, $\mathrm{cm}^{3}$ (sagittal MR images) & $149.54 \pm 19.90$ & $207.64 \pm 18.64$ & $0.028^{*}$ & $172.13 \pm 20.28$ & 0.505 \\
\hline
\end{tabular}

${ }^{\mathrm{a}}$ Statistical data are represented as mean \pm standard error of the mean (SEM)

${ }^{\mathrm{b}}$ Significant difference with normal group.

significant reduction after surgery $(\mathrm{P}<0.049)$. The result showed that the post-operative cerebellar volume in sagittal MR planes reduced compared with pre-operative volume, but this change was not statistically significant.

The data analysis of imaging is presented in Figure 2. The post-operative volume of the MB tumor was statistically reduced by more than $90 \%$ in both axial $(\mathrm{P}=0.046)$ and sagittal $(\mathrm{P}=0.028)$ planes in comparison to the preoperative volume. The pre-operative volume of the cerebellum in axial/sagittal planes increased by $38 \%$ in patients compared to the normal subjects.

\section{Discussion}

Primary tumor volume is an important prognostic factor that affects survival compared to the stages of the tumor (19). Some studies reported that calculation of the tumor volume with pre-catchment imaging methods may be useful to assess the surgical treatment options, and response of the tumor to the treatments such as radiation therapy, affects the anatomical structure of the brain $(20$, 21). Tumor volume is also used to determine tumor location, relationship with adjacent structures and its directions (22).

Volumetric measurement of the tumor area in mag- 
A

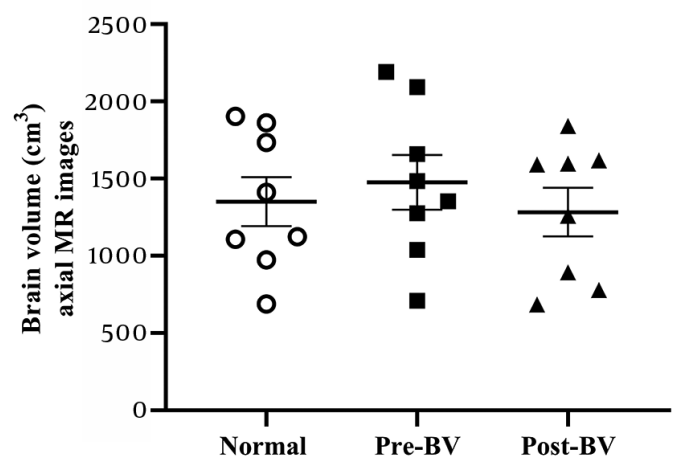

C

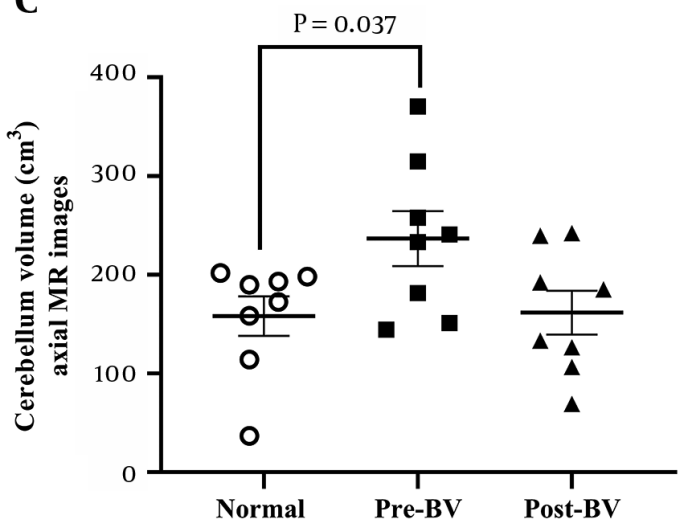

E

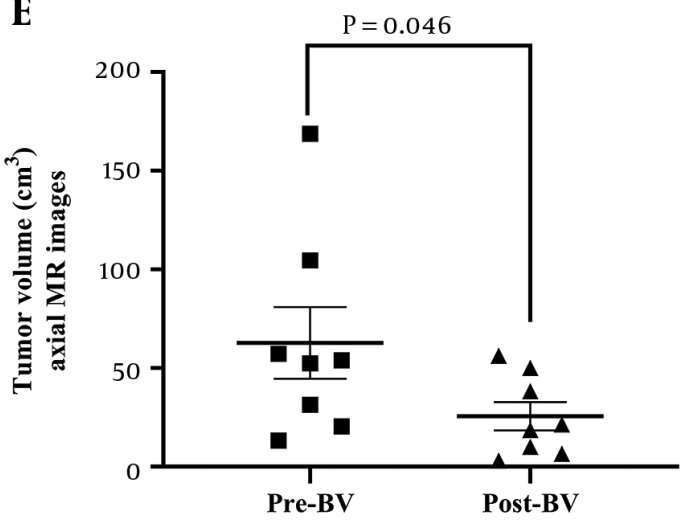

B

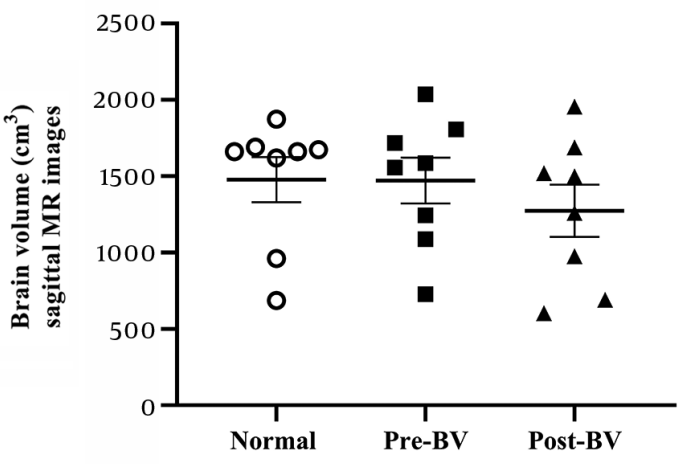

D

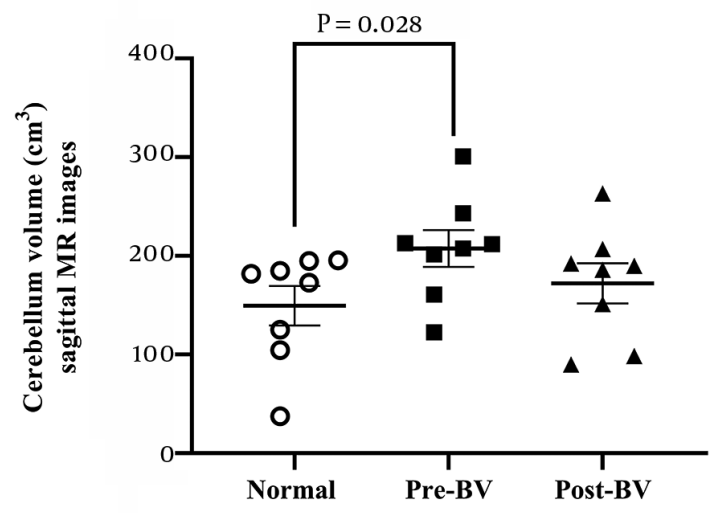

F

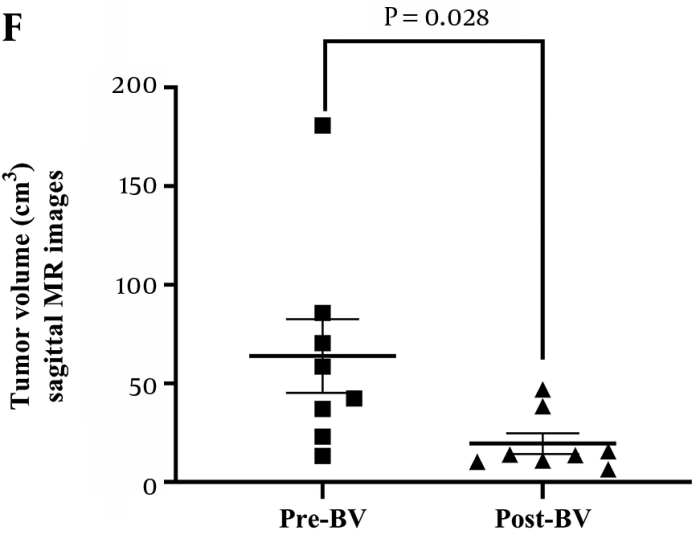

Figure 2. The volumes of the brain, cerebellum, and medulloblastoma tumor are represented in both axial and sagittal MR planes. Dot plots show the brain volume in the axial and sagittal planes (A and B), cerebellar volume in the axial and sagittal planes (C and D), and tumor volume in axial and sagittal planes (E and F) in the experimental groups. Each dot represents one subject. (BV: brain volume, CV: cerebellar volume, and TV: tumor volume).

netic resonance imaging (MRI) is clinically important for diagnosis and treatment assessment in patients with brain tumors.

Moreover, stereology is used to calculate unbiased properties of structures existing in three dimensions (3D) from two-dimensional (2D) medical images. An unbiased estimate of organ volume can be obtained using the Cavalieri principle, considered a stereological method (23). 
Table 2. Volume of the Brain, Cerebellum, and Medulloblastoma Tumor Represented in Both Axial and Sagittal MR Planes in the Pre- and Post- Operation Sessions in the Patient Group ${ }^{\mathrm{a}}$

\begin{tabular}{lccc}
\hline \multirow{2}{*}{ Parameters } & \multicolumn{3}{c}{ Patient group } \\
\cline { 2 - 4 } & Pre operation & Post operation & Pvalue \\
\hline $\begin{array}{l}\text { Brain volume, } \mathbf{c m}^{\mathbf{3}} \\
\text { (axial MR images) }\end{array}$ & $1475.62 \pm 177.17$ & $1282.52 \pm 157.09$ & 0.505 \\
\hline $\begin{array}{l}\text { Brain volume, } \mathbf{c m}^{3} \\
\text { (sagittal MR images) }\end{array}$ & $1471.14 \pm 150.17$ & $1273.91 \pm 170.37$ & 0.328 \\
\hline $\begin{array}{l}\text { Cerebellar volume, } \mathbf{c m}^{\mathbf{3}} \\
\text { (axial MR images) }\end{array}$ & $236.81 \pm 27.82$ & $161.74 \pm 22.22$ & $0.049^{\mathrm{b}}$ \\
$\begin{array}{l}\text { Cerebellar volume, } \mathbf{c m}^{\mathbf{3}} \\
\text { (sagittal MR images) }\end{array}$ & $207.64 \pm 18.64$ & $172.13 \pm 20.28$ & 0.130 \\
\hline $\begin{array}{l}\text { Tumor volume, } \mathbf{c m}^{\mathbf{3}} \\
\text { (axial MR images) }\end{array}$ & $62.76 \pm 18.13$ & $25.53 \pm 7.16$ & $0.046^{\mathrm{b}}$ \\
\hline $\begin{array}{l}\text { Tumor volume, } \mathbf{c m}^{\mathbf{3}} \\
\text { (sagittal MR images) }\end{array}$ & $63.91 \pm 18.71$ & $19.51 \pm 5.22$ & $0.028^{\mathrm{b}}$ \\
\hline
\end{tabular}

${ }^{\text {a }}$ Statistical data are represented as mean \pm standard error of the mean (SEM).

${ }^{\mathrm{b}}$ Significant difference between pre- and post- operation sessions

In fact, this method has the following advantages for the researcher: (1) The structure under study needs no preconditioning since the Cavalieri method is a design-based method but not a model based approach; (2) The actual properties of the structure, such as section thickness, are taken into consideration; and (3) The sampling or estimating system can be easily modified to obtain an appropriate coefficient of variation. Furthermore, the reliability and efficiency of the Cavalieri method for volume determination have been confirmed frequently (24).

Applying the Cavalieri principle in studies aimed at obtaining quantitative data on irregularly shaped threedimensional objects suggests benefits such as consequential quantitative data, application of strict sampling procedures, easily reproducible data, and a well-established theoretical background, making the reliability of the data easy for examination (25-27). Therefore, this method accompanied with MR imaging data can be potentially applied as a simple, reliable and quantitative method for diagnosis and follow-up of the treatment options in patients with brain tumors.

There has been growing recent interest in quantitative techniques for measuring volume using CT scans or MRI. Some proposed geometric methods for assessing the volumetric properties of radiological images based on direct geometric dimensions such as length, diameter, or the largest diameter measurements $(28,29)$. In such methods, it has been presumed that the structure under investigation has an ellipsoidal, spherical, or known geometric shape. However, the assumption of such smooth geometric properties in biological tissues is not always correct (23). In addition, the other techniques such as computer-based 3D volumetric reconstructions, and planimetry methods, are also used for volume estimations (30-33). The planimetric methodologies give more precise and accurate results compared to the above-mentioned geometric techniques $(28,30,31)$.

However, several studies showed that there are also assumptions in the planimetric methods resulting in volume measurement over-estimation and some degree of systematic bias $(28,29,34,35)$. In addition, the computerized reconstructions generally require quite expensive equipment and trained personnel for their routine application, and these features make these applications unsuitable for most clinics or research centers $(36,37)$.

Point counting techniques represent a more reliable and efficient approach than a planimetric methods (38-42); therefore, results obtained through point counting will be a direct and assumption-free estimate of the total volume of the interest structure, and hence very valuable in monitoring brain tumors.

In fact, the volume of any structure may be considered using histological sections, CT or MR images through Cavalieri method (22). Other advantages of this method are cost and time efficiency (43).

Results showed that the tumor volumes in axial/sagittal sections after surgery were significantly lower than those before surgery. In pediatric population, the spread of residual surgical resection has been shown to influence the survival outcomes. Excision of more than $90 \%$ of the tumor is correlated with improved survival rate in children older than three years (44). In our study, in four patients, more than $90 \%$ of the tumor was removed, suggesting an increase in survival rate. The results of the present study also showed differences in the volume of the cerebellum between the control and patient groups before surgery. According to this result, the cerebellum volume in axial and sagittal MRI images in patients with medulloblastoma tumor were significantly higher compared to that of the control group. This agreement with structural MR scanning studies revealed that medulloblastoma tumor can cause bilateral olivary hypertrophy in the human cerebellum which is known as hypertrophic olivary degeneration (HOD) (45).

A common problem in the stereological method is an over-projection or under-projection effect, an artifact that results from section thickness. This problem also exists when MRI or CT scans are used for volume estimations (23, $24,46)$. There are several solutions for management of the under-projection or over-projection effects of radiological imaging $(47,48)$. A previous study showed that the approach formulized as Equation 1 eliminates possible overestimation due to over-projection (24). Therefore, we used this method in the present study. 
Some brain tumors such as MB do not show a smooth demarcation with brain on MRI or CT scans. Therefore, it is almost impossible to obtain reliable quantitative data on tumor size using the diameter or length of the tumor on MRI. However, the surgeon needs the exact volume of the tumor for comparison of the pre-operative and postoperative scans (28).

During the operation, the whole tumor mass cannot be resected totally to avoid excision of the pathological structures with the surrounding healthy tissues. Thus, assessment of the exact tumor volume not only provides important data for the assessment of the size of the structures to be excised during the operation but also allows treatment planning and post-operative follow up $(28,34,49)$.

In addition, Cavalieri method using automated software is currently available and could be performed easily and rapidly. Stereological measurement can therefore provide additional useful data to supplement MR measurements, especially in borderline and controversial cases.

It could be concluded that the combination of MRI and the Cavalieri principle could estimate tumor volume that may be useful in evaluating the efficiency of surgical treatment and prognostication of tumor regression rate. The presented method does not require any change in routine procedures and can be performed on any complete set of MRI scans. The method can be efficiently used without any need for additional equipment and expert personnel that is required for routine MRI.

\section{Acknowledgments}

The authors would like to thank the managers and physicians of the Imaging Center of Namazi Hospital (Shiraz). Our special thanks go to staff members of imaging center for assistance in patient selection.

\section{Footnotes}

Authors' Contributions: Study concept and design: Farzaneh Dehghani, Seyede Fatemeh Hosseini; acquisition of data: Seyede Fatemeh Hosseini, Reza Jali, Narges Sotoudeh; analysis and interpretation of data: Seyede Fatemeh Hosseini; drafting of the manuscript: Seyede Fatemeh Hosseini; critical revision of the manuscript for important intellectual content: Reza Jalli, Sadegh Masoudi

Conflict of Interests: The authors declare no conflict of interests.

Ethical Approval: IR.SUMS.REC.1396.S661.

Funding/Support: Shiraz University of Medical Sciences supported this study.

\section{References}

1. Drevelegas A, Chourmouzi D, Constantinides M, Drevelegas K. Posterior Fossa Tumors. Journal of Pediatric Neuroradiology. 2016;5(2):89-110.

2. Felton K, Hogg A, Liang L, Aiken C, Klonisch T, van Landeghem F, et al. Primary Pediatric Brain Tumors of the Posterior Fossa: Part I. Development of the Cerebellum from Molecular Aspects to Diseases. Springer; 2017. p. 301-26.

3. Koustenis E, Driever PH, de Sonneville L, Rueckriegel SM. Executive function deficits in pediatric cerebellar tumor survivors. european journal of paediatric neurology. 2016;20(1):25-37.

4. Roth JJ, Fierst TM, Waanders AJ, Yimei L, Biegel JA, Santi M. Whole chromosome 7 gain predicts higher risk of recurrence in pediatric pilocytic astrocytomas independently from KIAA1549-BRAF fusion status. Journal of Neuropathology \& Experimental Neurology. 2016;75(4):306-15.

5. Sexton-Oates A, Dodgshun A, Hovestadt V, Jones DT, Ashley DM, Sullivan $\mathrm{M}$, et al. Methylation profiling of paediatric pilocytic astrocytoma reveals variants specifically associated with tumour location and predictive of recurrence. Molecular oncology. 2017.

6. Nobusawa S, Hirato J, Yokoo H. Molecular genetics of ependymomas and pediatric diffuse gliomas: a short review. Brain tumor pathology. 2014;31(4):229-33.

7. Cage TA, Clark AJ, Aranda D, Gupta N, Sun PP, Parsa AT, et al. A systematic review of treatment outcomes in pediatric patients with intracranial ependymomas: A review.Journal of Neurosurgery: Pediatrics. 2013;11(6):673-81.

8. Sinha P, Ahmad M, Varghese A, Parekh T, Ismail A, Chakrabarty A, et al. Atypical teratoid rhabdoid tumour of the spine: report of a case and literature review. European Spine Journal. 2015;24(4):472-84.

9. Slavc I, Chocholous M, Leiss U, Haberler C, Peyrl A, Azizi AA, et al. Atypical teratoid rhabdoid tumor: improved long-term survival with an intensive multimodal therapy and delayed radiotherapy. The Medical University of Vienna Experience 1992-2012. Cancer medicine. 2014;3(1):91-100.

10. Christopherson KM, Bradley JA, Rotondo RL, Pincus DW, Fort JA, Morris CG, et al. Local control in non-metastatic medulloblastoma. Acta Oncologica. 2014;53(9):1151-7.

11. Coluccia D, Figuereido C, Isik S, Smith C, Rutka JT. Medulloblastoma: tumor biology and relevance to treatment and prognosis paradigm. Current neurology and neuroscience reports. 2016;16(5):43.

12. DeSouza R, Jones BR, Lowis SP, Kurian KM. Pediatric medulloblastoma-update on molecular classification driving targeted therapies. Frontiers in oncology. 2014;4:176.

13. Kopecky AS, Khan AJ, Pan W, Drachtman R, Parikh RR. Outcomes and patterns of care in a nationwide cohort of pediatric medulloblastoma: Factors affecting proton therapy utilization. Adv Radiat Oncol. 2017;2(4):588-96. doi: 10.1016/j.adro.2017.07.007. [PubMed: 29204526]. [PubMed Central: PMCPmc5707421].

14. Sonmez OF, Odaci E, Bas O, Colakoglu S, Sahin B, Bilgic S, et al. A stereological study of MRI and the Cavalieri principle combined for diagnosis and monitoring of brain tumor volume. Journal of Clinical Neuroscience. 2010;17(12):1499-502.

15. Howard MA, Roberts N, Garciª-Fiñana M, Cowell PE. Volume estimation of prefrontal cortical subfields using MRI and stereology. Brain Research Protocols. 2003;10(3):125-38.

16. Gong QY, Eldridge PR, Brodbelt AR, Garcia-Finana M, Zaman A, Jones B, et al. Quantification of tumour response to radiotherapy. Br J Radiol. 2004;77(917):405-13. doi:10.1259/bjr/85294528. [PubMed: 15121704].

17. Liu J, Udupa JK, Odhner D, Hackney D, Moonis G. A system for brain tumor volume estimation via MR imaging and fuzzy connectedness. Comput Med Imaging Graph. 2005;29(1):21-34. doi: 10.1016/j.compmedimag.2004.07.008. [PubMed: 15710538]. 
18. Ertl-Wagner BB, Blume JD, Peck D, Udupa JK, Herman B, Levering A, et al. Reliability of tumor volume estimation from MR images in patients with malignant glioma. Results from the American College of Radiology Imaging Network (ACRIN) 6662 Trial. Eur Radiol. 2009;19(3):599-609. doi: 10.1007/s00330-008-1191-7. [PubMed: 18925402]. [PubMed Central: PMC2636854].

19. Bingol F, Yoruk O, Bingol BO, Erdemci B, Ozkan O, Mazlumoglu MR. Estimation of the efficacy of chemo-radiotherapy on tumor regression in the patients with laryngeal cancer via computerized tomography using the Cavalieri method. Acta oto-laryngologica. 2016;136(2):164-7.

20. Toussaint M, Pinel S, Auger F, Durieux N, Thomassin M, Thomas E, et al. Proton MR spectroscopy and diffusion MR imaging monitoring to predict tumor response to interstitial photodynamic therapy for glioblastoma. Theranostics. 2017;7(2):436.

21. Bainbridge H, Salem A, Tijssen RH, Dubec M, Wetscherek A, Van Es C, et al. Magnetic resonance imaging in precision radiation therapy for lung cancer. Translational lung cancer research. 2017;6(6):689.

22. Unal B, Kara A, Aksak S, Unal D. A stereological assessment method for estimating the surface area of cycloids. Eurasian J Med. 2010;42(2):6673. doi: 10.5152/eajm.2010.20. [PubMed: 25610126]. [PubMed Central: PMCPmc4261337].

23. Howard V, Reed M. Unbiased Stereology: Three-Dimensional Measurement in Microscopy. Taylor \& Francis; 2004.

24. Sahin B, Emirzeoglu M, Uzun A, Incesu L, Bek Y, Bilgic S, et al. Unbiased estimation of the liver volume by the Cavalieri principle using magnetic resonance images. Eur J Radiol. 2003;47(2):164-70. doi: 10.1016/s0720-048x(02)00152-3. [PubMed: 12880999].

25. Gundersen HJ, Jensen EB, Kieu K, Nielsen J. The efficiency of systematic sampling in stereology-reconsidered.J Microsc. 1999;193(Pt3):199-211. doi:10.1046/j.1365-2818.1999.00457.x. [PubMed:10348656].

26. Kubinova L, Janacek J, Ribaric S, CebasekV, Erzen I. Three-dimensional study of the capillary supply of skeletal muscle fibres using confocal microscopy. J Muscle Res Cell Motil. 2001;22(3):217-27. doi: 10.1023/a:1012201314440. [PubMed: 11763194].

27. Kubinova L, Janacek J. Confocal microscopy and stereology: estimating volume, number, surface area and length by virtual test probes applied to three-dimensional images. Microsc Res Tech. 2001;53(6):425-35. doi:10.1002/jemt.1112. [PubMed: 11525261].

28. Xue D, Albright RJ. Microcomputer-based technique for 3-D reconstruction and volume measurement of computed tomographic images. Comparison of geometric and planimetry post-operative tumor volume effects on patient survival. Comput Biol Med. 1999;29(6):37792. doi: 10.1016/s0010-4825(99)00016-5. [PubMed: 10591172].

29. Stocchetti N, Croci M, Spagnoli D, Gilardoni F, Resta F, Colombo A. Mass volume measurement in severe head injury: accuracy and feasibility of two pragmatic methods. J Neurol Neurosurg Psychiatry. 2000;68(1):14-7. doi: 10.1136/jnnp.68.1.14. [PubMed: 10601394]. [PubMed Central: PMC1760611].

30. Albright RJ, Fram EK. Microcomputer-based technique for 3-D reconstruction and volume measurement of computer tomographic images. Part 1: Phantom studies. Invest Radiol. 1988;23(12):881-5. doi: 10.1097/00004424-198812000-00001. [PubMed: 3203990].

31. Albright RJ, Fram EK. Microcomputer-based technique for 3-D reconstruction and volume measurement of computed tomographic images. Part 2: Anaplastic primary brain tumors. Invest Radiol. 1988;23(12):886-90. doi: 10.1097/00004424-198812000-00002. [PubMed: 2849594].

32. Chen MF, Hwang TL, Hung CF. Human liver regeneration after major hepatectomy. A study of liver volume by computed tomography. Ann Surg. 1991;213(3):227-9. doi:10.1097/00000658-199103000-00008. [PubMed: 1998403]. [PubMed Central: PMC1358332].

33. Blank M, Kalender WA. Medical volume exploration: gaining insights virtually. Eur J Radiol. 2000;33(3):161-9. doi: 10.1016/s0720048x(99)00140-0. [PubMed: 10699733].

34. Weltens C, Menten J, Feron M, Bellon E, Demaerel P, Maes F, et al. In- terobserver variations in gross tumor volume delineation of brain tumors on computed tomography and impact of magnetic resonance imaging. Radiother Oncol. 2001;60(1):49-59. doi: 10.1016/s01678140(01)00371-1. [PubMed: 11410304].

35. Rosen GD, Harry JD. Brain volume estimation from serial section measurements: a comparison of methodologies. J Neurosci Methods. 1990;35(2):115-24. doi: 10.1016/0165-0270(90)90101-k. [PubMed: 2283883].

36. Black KJ. On the efficiency of stereologic volumetry as commonly implemented for three-dimensional digital images. Psychiatry Res. 1999;90(1):55-64. doi: 10.1016/s0925-4927(99)00003-7. [PubMed: 10320211].

37. Clatterbuck RE, Sipos EP. The efficient calculation of neurosurgically relevant volumes from computed tomographic scans using Cavalieri's Direct Estimator. Neurosurgery. 1997;40(2):339-42. discussion 343. doi: 10.1097/00006123-199702000-00019. [PubMed: 9007867]

38. Gundersen HJG, Boysen M, Reith A. Comparison of semiautomatic digitizer-tablet and simple point counting performance in morphometry. Virchows Archiv B. 1981;37(1):317-25. doi: 10.1007/BF02892580.

39. Mathieu O, Cruz-Orive LM, Hoppeler H, Weibel ER. Measuring error and sampling variation in stereology: comparison of the efficiency of various methods for planar image analysis. J Microsc. 1981;121(Pt 1):7588. doi: 10.1111/j.1365-2818.1981.tb01200.x. [PubMed: 7014911].

40. Sahin B, Mazonakis M, Akan H, Kaplan S, Bek Y. Dependence of computed tomography volume measurements upon section thickness: an application to human dry skulls. Clin Anat. 2008;21(6):479-85. doi: 10.1002/ca.20664. [PubMed: 18627101].

41. Sahin B, Acer N, Sonmez OF, Emirzeoglu M, Basaloglu H, Uzun A, et al. Comparison of four methods for the estimation of intracranial volume: a gold standard study. Clin Anat. 2007;20(7):766-73. doi: 10.1002/ca.20520. [PubMed: 17708568].

42. Varoglu AO, Odaci E, Gumus H, Keles ON, Unal B, Deniz O. Evaluation of patients with multiple sclerosis using a combination of morphometrical features and clinical scores. J Clin Neurosci. 2010;17(2):191-5. doi: 10.1016/j.jocn.2009.04.023. [PubMed: 20036126].

43. Sonmez OF, Temel Y, Visser-Vandewalle V, Sahin B, Odaci E. A new evaluation method for the intracranial volume changes and subdural effusion of patients following endoscopic third ventriculostomy. Clin Neurol Neurosurg. 2013;115(2):160-4. doi: 10.1016/j.clineuro.2012.04.023. [PubMed: 22613878].

44. Sivasankaran A, Sgouros S, Walsh R, Hockley A. Medulloblastoma in children: Birmingham experience. Journal of Pediatric Neurosciences. 2006;1(2):49.

45. Sanverdi SE, Oguz KK, Haliloglu G. Hypertrophic olivary degeneration in children: four new cases and a review of the literature with an emphasis on the MRI findings. The British journal of radiology. 2012;85(1013):511-6.

46. Gadeberg P, Gundersen HJ, Taagehoj F, Jakobsen J. MRI volume measurements of hypointense objects. A phantom study using stereological methods. J Neurosci Methods. 2002;114(2):149-57. doi: 10.1016/s01650270(01)00513-1. [PubMed: 11856565].

47. Cruz-Orive LM. Particle number can be estimated using a disector of unknown thickness: the selector. J Microsc. 1987;145(Pt 2):121-42. [PubMed: 3553604].

48. Pakkenberg B, Boesen J, Albeck M, Gjerris F. Unbiased and efficient estimation of total ventricular volume of the brain obtained from CTscans by a stereological method. Neuroradiology.1989;31(5):413-7. doi: 10.1007/bfo0343866. [PubMed: 2594185].

49. Lopez-Aguilar E, Sepulveda-Vildosola AC, Rivera-Marquez $\mathrm{H}$, Cerecedo-Diaz F, Hernandez-Contreras I, Ramon-Garcia G, et al. Preirradiation ifosfamide, carboplatin, and etoposide for the treatment of anaplastic astrocytomas and glioblastoma multiforme: a phase II study. Arch Med Res. 2000;31(2):186-90. doi: 10.1016/s01884409(00)00051-5. [PubMed: 10880725]. 